\title{
Author Index to Volume 35
}

Adamson M, 197
Aladjem M, 372
Anderson DF, 555
Anderson PAW, 550
Andrew M, 78
Aoki T, 598
Aono S, 629
Arant Jr BS, 671
Arar M, 474
Ardito T, 152
Ascuitto RJ, 244
Atiyeh BA, 671
Axelrod R, 470
Bada HS, 565
Bailie KEM, 164
Bair DK, 560
Baker L, 84
Ballin A, 682
Bancalari E, 316, 536
Barefoot TK, 633
Baroncelli GI, 409
Barr J, 682
Barrett H, 690
Barrington KJ, 15
Baum M, 447, 474
Becker K, 307
Beitins IZ, 218
Bell A, 649
Bennett SH, 62
Benson L, 78
Berger M, 68
Bernard G, 640
Berry GT, 141
Bertelloni S, 409
Birdsey TJ, 376
Bistritzer T, 372
Blanchette V, 78
Bloch KD, 523
Block SM, 424
Boesiger P, 431
Boon AW, 677
Borondy M, 218
Bourre J-M, 640
Brand R, 541
Brandtzaeg P, 625
Brantly ML, 197
Brasel JA, 223
Bridges JM, 164
Bridonneau C, 696
Briggs KK, 404
Brill-Edwards P, 78
Brown WR, 424
Bruhn H, 614
Bry K, 130
Buchina ES, 633
Buchli R, 431
Bundy M, 214
Burke JF, 580
Burns DK, 436
Burrows P, 78
Busija DW, 565
Butler M, 510
Calder NA, 321, 677
Campbell DE, 84
Carbone GMR, 41
Carisson B, 307
Carrascosa A, 362
Castillo L, 580
Chabanet C, 696
Chace DH, 96

Challa VR, 424

Chanez C, 640

Chan WWK, 15

Chapman TE, 580

Chen $Y, 233$

Christensen B, 3

Christensen RD, 169, 303

Chu RC, 3

Cinquanta L, 409

Clairambault J, 500

Cone EJ, 339

Cooper DM, 223

Corbett RJT, 436

Corkill JE, 299

Cosyns M, 293

Cotariu D, 372

Cristiano F, 188

Cruz MLA, 135

Curzi-Dascalova L, 500

Darville T, 397

Dasuki M, 510

David M, 78

Davidsson L, 117

Davis JM, 37

Davis LE, 555

Dawson CA, 20, 25

de Haan JB, 188

De Jong MCJW, 367

Dell RB, 704

del Rio L, 362

De Muinck Keizer-Schrama SMPF, 367

de Ridder MAJ, 367

Desgres J. 357

Deveney J, 299

de Vries LS, 148

de Winter JP, 54

Diamond G, 451

DiNero G, 409

Domenech FM, 362

Donahoe PK, 523

Donato L, 637

Donkerwolcke RA, 367

Douglas SD, 84

Drop SLS, 367

Dryja D, 690

Duara S, 536

Duffy LC, 690

Dvorák B, 348

Edberg KE, 571

Efrati $Y, 682$

Egberts J, 541

Eiselt M, 500

Ekstrand J, 157

Elliott SR, 729

Emancipator SN, 68

Eshel G, 682

Etches PC, 15

Evans DH, 50

Evans S, 372

Fair EC, 550

Feldhoff RC, 389

Ferrarri F, 50

Fineman JR, 664

Finer NN, 15

Fish W, 571

Fomon SJ, 157

Foster CM, 218

Fox RE, 589

Frahm J, 614
Frank L, 233, 530

Freiberg R, 383

Fujisawa T, 179

Fujita I, 255

Gahl WA, 197

Garcia D, 436

Gargosky SE, 720

Garland HO, 376

Gaudio KM, 152

George-Nascimento C, 30

Georgi M, 617

Gerhardt T, 536

Geske RS, 311

Gest AL, 560

Ghishan FK, 510

Gil A, 112

Ginsberg J, 78

Giralt M, 91

Glazier JD, 376

Gleason CA, 339

Gluckman PD, 657

Glueck CJ, 383, 602

Glueck HI, 383

Goetzman BW, 30

Goin JE, 451

Goldberg M, 372

Gonenne A, 37

Gorman A, 550

Gouyon JB, 357

Graham AJ, 15

Greenfield D, 383

Griffiths E, 690

Groenendaal F, 148

Guerra FA, 484

Gunn AJ, 657

Gusinyé $M, 362$

Hachey DL, 135

Haddad J, 637

Hahn SH, 197

Hallman M. 130

Hamada $Y, 10$

Hambidge KM, 404

Hamburg DC, 311

Hamer T, 383, 602

Hamilton JR, 45

Hanefeld F, 614

Hansen TN, 311

Hanson MA, 321, 677

Harada K, 506

Hardcastle J, 354

Hardcastle PT, 354

Hart CA. 299

Hattori $\mathrm{H}, 10$

Haws RM, 671

Hayakawa T, 10

Hayde M, 713

Hayward A, 293

Heaf D, 299

Heales SJR, 96

Hehre D, 316, 536

Heikinheimo M, 205

Heird WC, 704

Hellerqvist C, 571

Heymann MA, 664

Hipps R, 649

Hoath SB, 443

Hodson WA, 238

Hoffmann GF, 307

Hoffpauir JM, 389

Hokken-Koelega ACS, 367
Holmberg C, 205

Holt J, 184

Hopwood NJ, 218

Hoving EB, 461

Howes A, 171, 416

Huang J, 316

Hudault S, 696

Hurrell RF, 117

Husain SM, 376

Iglesias $\mathrm{R}, 91$

Ikegami M, 479, 685

Irvine $\mathrm{AE}, 164$

Jackson JC, 238

Jacobs RF, 397

Jansen GH, 148

Jensen S, 223

Jobe AH, 479, 685

Johansen HT, 184

Jones Jr MD, 339

Juif JG, 637

Juul SE, 238

Käär M-L, 594

Kaartinen JM, 594

Kahler SG, 96

Kahn A, 383

Kaler SG, 197

Kamei A, 334

Kashgarian M, 152

Kashyap S, 704

Kastenmayer P, 117

Kauffman KS, 515

Kauffmann F, 500

Kawase H, 179

Kawchak DA, 45!

Keino H, 629

Kelsall AWR, 50

Kernan WJ, 171

Kerrigan JR, 102

Kikkawa Y, 255

Killam AP, 550

Kilpatrick L, 84

Kingwell KG, 589

Kinsella MG, 238

Kirpalani H, 494

Kitterman JA, 484

Klautz RJM, 329

Klein PD, 135

Kletter GB, 218

Kleuber KM, 389

Knobel S, 510

Kohl S, 289

Koiwai O, 629

Kola I, 188

Koldovský O, 348

Konstan MW, 68

Koo H-C, 37

Kopelman H, 45

Korchak HM, 84

Korones SB, 565

Krebs NF, 404

Kruse B, 614

Kuitunen OOM, 344

Kuller FE, 602

Kumar P, 321, 677

Kuusela P, 205

Kydon DW, 244

Lahat E, 682

Landers DV, 289

Landy H, 416 
Langermans JAM, 725

Lappalainen U, 130

Laptook AR, 436

Larsson A, 307

Lassiter HA, 389

Lee C-CH, 484

Lefauconnier J-M, 640

Leffler CW, 565

Lehrnbecher T, 720

Levi M, 474

Lewis JF, 479

Liechty KW, 303

Linehan $\mathrm{JH}, 25$

Lister G, 1

Livne A, 682

Li Y, 169

Lohr JL, 325

London ED, 339

Lönnerdal B, 117

Lowe LS, 713

Lozon M, 649

Lushkov G, 682

Mack DR, 209

MacLeod RJ, 45

Maitre M, 637

Mallard EC, 657

Mampel T, 91

Marchini JS, 580

Mark J, 637

Markovs ME, 218

Martin E, 431

Martin J, 470

Marzinotto V, 78

Massicotte P, 78

Mayatepek E, 307

McCawley LJ, 84

McClure BG, 164

Meadow W, 649

Médigue C, 500

Merrill DC, 250

Merritt TA, 30

Merth IT, 541

Messer J, 637

Mikawa H, 10

Milam T, 289

Millington DS, 96

Milstein JM, 62

Mimouni F, 135

Mimura S, 629

Mollnes TE, 184

Moltó L, 112

Montgomery CA, 311

Moody DM, 424

Morillas J, 112

Moromisato D, 223

Moromisato M, 223

Morrison JA, 602

Mortola JP, 490

Morton MJ, 325

Moshin J, 20

Mousson C, 357

Mughal MZ, 376

Mullenix PJ, 171, 416

Müller HL, 720

Muskiet FAJ, 461

Nakagawa T, 629

Nassar R, 550

Nelin LD, 20, 25

Nelson SE, 157

Neumann AW, 209

Nexø E, 107

Nielsen EW, 184

Nijeboer HJ, 461

Norvell TM, 68

O'Brien TP, 339
Ogra PL, 690

Oh Y, 720

Ohashi T, 685

Ohisalo JJ, 594

Ohls RK, 169, 303

Okah FA, 443

Oliver C, 197

Onishi S, 629

Ozaki T, 334

Parker Jr CR, 633

Parker RA, 571

Pearce WJ, 729

Peirano P, 500

Phillips J, 510

Pickoff AS, 55, 244

Pinkerton K, 685

Pitkänen S, 205

Plopper CG, 30

Pohlandt F, 125, 701

Polak MJ, 228

Policova Z, 209

Polk D, 479

Pollak A, 713

Pompa K, 443

Pons $F, 362$

Poon A, 78

Poulsen SS, 107

Pourcyrous M, 565

Prantner JE, 141

Pratt J, 299

Quanjer PH, 541

Raaberg L, 107

Rabin O, 640

Raibaud P, 696

Ramage D, 244

Ramakrishnan R, 704

Randle C, 649

Read LC, 30

Reller MD, 325, 555

Rennie JM, 50

Rickaby DA, 25

Riepenhoff-Talty M, 690

Rios R, 55

Robillard JE, 250

Robles R, 112

Rodriguez-Pierce M, 530

Rodriguez R, 602

Roe CR, 96

Roe DS, 96

Rognum TO, 625

Rogol AD, 102

Roman C, 329

Rose JC, 41

Rosenblatt DS, 3

Rosenfeld RG, 720

Rosenfeld WN, 37

Ross-Ascuitto NT, 244

Rossman J, 690

Rudinsky B, 649

Rudolph AM, 329

Ruffin D, 690

Rumpel H, 431

Rupp A, 611

Ryan JP, 470

Saggese G, 409

Saiki C, 490

Sakarcan A, 447

Sallan SE, 171

Salo MK, 205

Sanchez M, 580

Sanchez-Pescador L, 289

Sánchez-Pozo A, 112

Sandberg K, 571

Sarnesto A, 344
Sasaoka Y, 629

Sasidharan P, 20

Sato H, 629

Sauer PJJ, 1

Savich RD, 484

Savilahti E, 344

Sayahtaheri-Altaie S, 690

Scanlin TF, 451

Schaefer F, 617

Schaller P, 494

Schärer K, 617

Schibler KR, 303

Schmidt E, 611

Schreiber JR, 68

Schulze A, 494

Schulze KF, 704

Schunior A, 171, 416

Schwarzenberg SJ, 214

Segar JL, 250

Seidler FJ, 515

Seikaly MG, 671

Sekine I, 179

Setchell KDR, 135

Shaul PW, 671

Shears P, 299

Sherman PM, 209

Shiota T, 506

Sibley CP, 376

Siegel NJ, 152

Sieve-Smith L, 602

Silberbach M, 555

Simell O, 205

Simpson K, 397

Sindhu RK, 255

Slotkin TA, 515

Smith BA, 250

Smith JP, 289

Smyth A, 299

Smyth J, 677

Soliz A, 316

Sosa F, 602

Sosa G, 62

Sosenko IRS, 530

Sparling K, 78

Spassov L, 500

Stallings VS, 451

Standaert TA, 238

Stanley CA, 84

States B, 141

St. Clair DK, 41

Steenwijk TM, 725

Sterett R, 436

Stevenson DK, 713

Stolfi A, 55, 244

Stroop D, 383

Styne D, 30

Suen HC, 523

Suguihara C, 316, 536

Sundell $H, 571$

Suzuki M, 598

Sweezey NB, 45

Tabor BL, 479

Tabor D, 397

Takada G, 506

Takahashi Y, 506

Takashima S, 334

Takeshita S, 179

Tamura M, 506

Tan WKM, 657

Tarantal AF, 30

Tarbell NJ, 171, 416

Taylor CJ, 354

Taylor DA, 228

Teitel DF, 329

Teramo K, 130

Thomas CJ, 20

Thornburg KL, 325

Thornton PS, 84

Thrane PS, 625

Thulesen J, 107

Thulin G, 152

Tildon JT, 589

Timmons C, 447

Tollefsbol G, 436

Tomezsko JL, 451

Tonoki H, 311

Töpfer A, 494

Tosi MF, 68

Townsend SF, 404

Toyono M, 506

Tracy T, 383

Trautman MS, 169

Traystman RJ, 339

Truog WE, 238

Tsang RC, 135

Tuca A, 91

Tymms MJ, 188

Ueland PM, 3

van Bel F, 329, 541

van Beusekom CM, 461

van der Grond J, 148

Vander Straten MC, 560

van Furth $A M, 725$

van Furth R, 725

Van Hove JLK, 96

Van Zaal MAE, 367

Veenhoven RH, 148

Veldhuis JD, 102

Vial MF, 696

Vilge $V, 637$

Villarroya F, 91

Viñas 0,91

Vinograd I, 682

Vreman HJ, 713

Waber DP, 171

Waffarn F, 130

Walker CK, 289

Wang SN, 55, 244

Weglage $\mathrm{J}, 611$

Weiner CP, 713

Welty SE, 311

Whitney P, 530

Whitney PL, 233

Whitsett JA, 30

Wickett RR, 443

Widness JA, 713

Williams BA, 321, 677

Williams CE, 657

Williams W, 78

Wilson JL, 389

Wilson KF, 720

Witkamp TD, 148

Wolff ED, 367

Wong J, 664

Wong WW, 135

Wu DE, 325

$\mathrm{Xu} Y A, 41$

Yamada Y, 629

Yamamoto M, 179

Yandrasitz JR, 14!

Yeste D, 362 